\title{
Viable spermatozoa in the bladder after electroejaculation of lion-tailed macaques (Macaca silenus)
}

\author{
N. E. Schaffer*§, M. Cranfield*, A. T. Fazleabas $†$ and R. S. Jeyendran $\ddagger$ \\ ${ }^{*}$ Baltimore Zoo, Druid Hill Park, Baltimore, MD 21217, USA; + Department of Obstetrics and \\ Gynecology, University of Illinois Medical Center, 840 S. Wood St., Chicago, IL 60612, USA; and \\ $\ddagger$ Institute of Reproductive Medicine, I11N. Wabash Avenue, Chicago, IL 60602, USA
}

\begin{abstract}
Summary. The bladder of 6 lion-tailed macaques was emptied and flushed with sterile saline. TALP-Hepes buffer was infused and the animals were electroejaculated. After electroejaculation, the semen quality was determined in the ejaculate and the bladder infusate. Of the 15 ejaculates analysed, a mean ( \pm s.e.m.) sperm count of $133.8( \pm 30.7) \times 10^{6}$ with $69.5( \pm 6.0) \%$ motility was obtained in the infusate as compared to the sperm count of $72.4( \pm 38.6) \times 10^{6}$ with significantly lower $(47 \cdot 7 \pm 5 \cdot 8 \%)$ motility in the ejaculate.
\end{abstract}

Keywords: macaque; retroejaculation; ejaculation; sperm viability

\section{Introduction}

Rectal probe electroejaculation is routinely used to obtain semen from non-human primates. This procedure has been reported to induce retrograde flow of semen into the bladder in many species. This loss of spermatozoa to the bladder may have a significant effect on sperm count. This study attempts to determine whether retrograde flow of semen occurs following electroejaculation in liontailed macaques, and if so, to estimate the relative loss of spermatozoa to the bladder and to study the feasibility of recovering these spermatozoa.

\section{Materials and Methods}

Animals. Six healthy lion-tailed macaques were placed in lateral recumbancy after sedation with $25 \mathrm{mg}$ ketamine $\mathrm{HCl} / \mathrm{kg}$ (Ketaset, Bristol Laboratories, Syracuse, NY, USA) intramuscularly. The bladder was emptied of urine by using a size 8 french catheter and subsequently rinsed with $20 \mathrm{ml}$ saline. Immediately, $20 \mathrm{ml}$ TALP-Hepes (Bavister et al., 1983) medium were infused into the bladder, the catheter removed and the animal electroejaculated.

Electroejaculation. A $2.5-\mathrm{cm}$ diameter rectal probe with three $130 \times 3 \mathrm{~cm}$ electrodes that were $0.7 \mathrm{~cm}$ apart was placed in the pelvic rectum with electrodes positioned ventrally. An electrical current of $20 \mathrm{~Hz}$ was delivered to the probe by a Lane P-3 Z stimulator (Lane Manufacturing Co., Denver, CO, USA). The pattern of electrical delivery consisted of rhythmic pulse stimulations at a constant voltage. The stimulus was applied in pulses of 3-sec duration with a $1-\mathrm{sec}$ rest between pulses. Three series of 10 pulses each, took place at settings of $5,8,10 \mathrm{~V}$. Stimuli were applied at a current density which did not exceed $0.25 \mathrm{~mA} / \mathrm{mm}^{2}$ (Gould et al., 1978). The probes were adjusted inside the rectum to obtain maximal erection of the penis. After each series, the urethra was 'stripped' (massaged via the rectum) to remove seminal fluid from the urethra (Roussel \& Austin, 1968).

After electroejaculation, the bladder was catheterized again and the contents emptied. The volume, sperm count and motility were determined in the bladder infusate and ejaculate after electroejaculation.

The mean and standard error of the mean were calculated and paired $t$ tests were performed to assess the significant differences in means of sperm count and motility between the ejaculate and the bladder infusate.

§resent address: Lincoln Park Zoo, 2200 North Cannon Drive, Chicago, IL 60614, USA. 


\section{Results}

No infections occurred after the procedure even though some attempts resulted in the presence of blood in the catheter. Spermatozoa were not found in the urine or saline lavages before electroejaculation in any of the animals. After electroejaculation, spermatozoa were found in all but one of the bladder infusates (Table 1). The volume of the bladder samples was significantly higher because of the inclusion of $20 \mathrm{ml}$ medium. The lack of significant difference between sperm numbers in the ejaculate and the bladder infusate indicated that at least half of the spermatozoa flowed into the bladder during electroejaculation.

Table 1 also demonstrates a significant $(P<0.05)$ improvement in the motility of spermatozoa obtained from the bladder infusate. However, raw samples were incubated in seminal fluids for 30 min which may be detrimental to sperm viability and the TALP-Hepes medium may improve sperm motility.

Table 1. Semen parameters of ejaculate and bladder infusates obtained from 6 lion-tailed macaques after electroejaculation (15 samples)

\begin{tabular}{lccc}
\hline & $\begin{array}{c}\text { Volume } \\
(\mathrm{ml})\end{array}$ & $\begin{array}{c}\text { Sperm count } \\
\left(\times 10^{-6}\right)\end{array}$ & $\begin{array}{c}\text { Sperm motility } \\
(\%)\end{array}$ \\
\hline Ejaculate & $0 \cdot 7 \pm 0 \cdot 2$ & $72 \cdot 4 \pm 38 \cdot 6$ & $47 \cdot 7 \pm 5 \cdot 8$ \\
Bladder infusate & $23 \cdot 9 \pm 0 \cdot 8^{*}$ & $133 \cdot 8 \pm 30 \cdot 7$ & $69 \cdot 5 \pm 6 \cdot 0^{*}$ \\
\hline
\end{tabular}

Values are mean \pm s.e.m.

${ }^{*} P<0.05$ compared with value for ejaculate.

\section{Discussion}

Normal ejaculation is a neurologically sequenced event proceeding first with seminal emission into the posterior urethra and then with forceful propulsion of semen from the posterior urethra out of the distal penis by contraction of penile musculature (Rose, 1953; Kedia \& Markland, 1976). During this process external and internal sphincter synchrony along with proper peristaltic flow is required to encourage semen in an antegrade direction (Schienen \& Hupe, 1976; Newman et al., 1982; Thomas, 1983) as demonstrated by radiological studies of rams (Hovell et al., 1969) and marsupials (Rodger \& Pollitt, 1981). The lack of synchronous flow therefore traps semen in the posterior urethra and allows it to flow back into the bladder when stimulation ceases. Men that can ejaculate, but are partly dysfunctional will also retroejaculate (Reiser, 1961; Rose, 1953; Okamura et al., 1985). In addition, spermatozoa are also found in the bladders of paraplegic men after electroejaculation (Brindley, 1981; Sarkarati et al., 1987). The neurological dys-synchronous stimulation occurring after injury, or in the process of electroejaculation procedures, causes physiological disruption of normal ejaculation events and semen flows retrogradely into the bladder.

Preliminary cystocentesis studies have suggested loss of $60-80 \%$ of the total spermatozoa to the bladders of macaques (Schaffer et al., 1989). In the present study, on the average, $65 \%$ of the spermatozoa were lost to the bladder. Extensive loss of spermatozoa to the bladder during electroejaculation has been reported for many species of animals (Pineda et al., 1987). Cystocentesis studies by Dooley et al. $(1984,1986)$ determined a $6-61 \%$ and a mean $21 \%$ loss of spermatozoa to the bladder of electroejaculated cats and bulls, respectively.

Reports on retrograde sperm loss in man rarely give sperm counts. Only a few men exhibit complete retroejaculation, while the large majority have both retrograde and antegrade ejaculation. 
Analysis of available data (Halstead et al., 1987) from three retroejaculatory patients reported loss of $3-50 \%$ total sperm numbers to the bladder.

In the present study, pushing of spermatozoa back into the bladder by the catheter was a possibility, but 'stripping' was used to clear the semen from the urethral tract before introducing the catheter (Roussel \& Austin, 1968; Halstead et al., 1987) and any residual semen in the tract could not have produced the significant numbers of spermatozoa found in the bladder. In addition, no spermatozoa were found in the bladder infusate of one collection that produced significant numbers of spermatozoa in the ejaculated sample, indicating that 'stripping' the urethra per rectum was an effective means of removing semen from the urethra.

Total sperm recovery is needed for proper fertility determination and to provide greater numbers of spermatozoa for artificial manipulations. Other researchers have made attempts to prevent retroejaculation during electrostimulation. Brindley (1981) suggests that more appropriate electrode placement facilitates antegrade flow, while Warner et al. (1986) unsuccessfully blocked the neck of the bladder with a foley catheter. Most reports advocate retrieving spermatozoa from the bladder after alleviating the harmful effects of bladder contents by alkalizing the urine (Garcea et al., 1982; Cameron \& Gillett, 1985; Brassesco et al., 1988) or replacing it with medium. Bennett et al. (1987) replaced the bladder contents with a buffered medium before ejaculation and recovered bladder spermatozoa for artificial insemination. They were able to obtain successful pregnancies with this semen. With the lion-tailed macaque, replacing bladder contents with medium was used because of the difficulty in administering alkalizing drugs and the risk of drug interactions. Improved motility was seen in the spermatozoa in the TALP-Hepes medium recovered from the bladder in stimulated macaques. Spermatozoa recovered from the bladder have successfully fertilized eggs in vitro, and 2 pregnancies have resulted from bladder recovered spermatozoa (N. E. Schaffer, unpublished data).

\section{References}

Bavister, B.D., Leibfried, M.L. \& Lieberman, G. (1983) Development of pre-implantation embryos of the Golden hamster in a defined culture medium. Biol. Reprod. 28, 235-247.

Bennett, C.J., Ayers, J.W.T., Randolph, J.F., Seager, S.W.J., McCabe, M., Moinipanah, R. \& McGuire, E.J. (1987) Electroejaculation of paraplegic males followed by pregnancies. Fert. Steril. 48, 1070-1072.

Brassesco, M., Viscasillas, P., Burrel, L., Calab, J., Rajmil, O., Serra, J.M.P. \& Fargas, F.M. (1988) Sperm recuperation and cervical insemination in retrograde ejaculation. Fert. Steril. 49, 923-925.

Brindley, G.S. (1981) Electroejaculation: Its technique, neurological implications and uses. J. Neurol. Neurosurg. Psych. 44, 9-18.

Cameron, M.C. \& Gillett, W.R. (1985) The recovery of sperm, insemination and pregnancy in the treatment of infertility because of retrograde ejaculation. Fert. Steril. 44, 844 845 .

Dooley, M.P., Pineda, M.H., Hooper, J.G. \& Hsu, W.H. (1984) Retrograde flow of semen caused by electroejaculation in the domestic cat. Proc. 10th Int. Congr. Anim. Reprod. \& A. I., Urbana/Champaign 3, Abstr. 363.

Dooley, M.P., Pineda, M.H., Maurer, R.R. \& Lunstra, D.D. (1986) Evidence for retrograde flow of spermatozoa into the urinary bladder of bulls during electroejaculation. Theriogenology 26, 101-109.

Garcea, N., Caruso, A., Campo, S. \& Siccardi, P. (1982) Retrograde ejaculation: a more convenient method for artificial insemination. Eur. J. Obstet. Gynec. Reprod. Biol. 14, 175-178.

Gould, K.G., Warner, H. \& Martin, D.E. (1978) Rectal probe electroejaculation of primates. J. med. Primatol. 7, 213-222.

Halstead, L.S., VerVoort, S. \& Seager, S.W.J. (1987) Rectal probe electro-stimulation in the treatment of anejaculatory spinal cord injured men. Paraplegia 25, $120-129$.

Hovell, G.J.R., Ardran, G.M. \& Essenhigh, D.M. (1969) Radiological observations on electrically induced ejaculation in the ram. J. Reprod. Fert. 20, 383-388.

Kedia, K.R. \& Markland, C. (1976) The ejaculatory process. In Human Semen and Fertility Regulation in Men, pp. 497-503. Ed. E. S. E. Hafez. C. V. Mosby Co., St Louis.

Newman, H.F., Harry, R. \& Northrup, J.D. (1982) Physical basis of emission, ejaculation, and orgasm in the male urology. J. Urol 19, 341-349.

Okamura, K., Hirao, Y., Momose, H., Okajima, E. \& Yamada, K. (1985) Retrograde ejaculation caused by incomplete paralysis of the pelvic nerve. Urology. 25, 485-489.

Pineda, M.H., Dooley, M.P., Hembrough, F.B. \& Hsu, W.H. (1987) Retrograde flow of spermatozoa into the urinary bladder of rams. Am. J. vet. Res. 48, 562-568.

Rieser, C.H. (1961) The etiology of retrograde ejaculation and a method for insemination. Fert. Steril. 12, 488-492. 
Rodger, J.C. \& Pollitt, C.C. (1981) Radiographic examination of electroejaculation in marsupials. Biol. Reprod. 24, 1125-1134.

Rose, S.S. (1953) Investigation into sterility after lumbar ganglionectomy. Br. med. J. I, 247-250.

Roussel, J.D. \& Austin, C.R. (1968) Improved electroejaculation of primates. J. Inst. Anim. Tech. 19, 22-32.

Sarkarati, M., Rossier, A.B. \& Fam, B.A. (1987) Experience in vibratory and electro-ejaculation techniques in spinal cord injury patients: a preliminary report. $J$. Urol. 138, 59-62.
Schaffer, N.E., Cranfield, M., Meehan, T. \& Kempske, S. (1989) Semen collection and analysis in the conservation of endangered non human primates. Zoo Biol., Suppl. 1, 47-60.

Schienen, C. \& Hupe, H. (1976) Retrograde ejaculation. Urology 16, 108-110.

Thomas, A.J. (1983) Ejaculatory disfunction. Fert. Steril. 39, 445-449.

Warner, H., Martin, D.E., Perkash, I., Speck, V. \& Nathan, B. (1986) Electrostimulation of erection and ejaculation and collection of semen in spinal cord injured humans. J. Rehab. Res. Dev. 23, 21-31.

Received 9 February 1989 